\title{
Amelioration of nerve conduction velocity following simultaneous kidney/pancreas transplantation is due to the glycaemic control provided by the pancreas
}

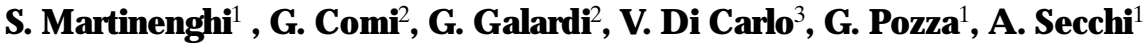 \\ ${ }^{1}$ Department of Medicine, University of Milan, San Raffaele Scientific Institute, Milan, Italy \\ ${ }^{2}$ Department of Neurology, University of Milan, San Raffaele Scientific Institute, Milan, Italy \\ ${ }^{3}$ Department of Surgery, University of Milan, San Raffaele Scientific Institute, Milan, Italy
}

Summary Diabetic polyneuropathy is a common, disabling chronic complication of diabetes mellitus. Previous studies have suggested that combined pancreas-kidney transplantation can ameliorate nerve conduction. The relative contribution of the correction of hyperglycaemia and uraemia on nerve function is still a matter of debate. Nerve conduction velocity (NCV) was assessed before and after simultaneous pancreas and kidney transplantation, and before and after pancreas graft failure in five insulindependent diabetic (IDDM) patients affected by severe diabetic polyneuropathy. Sensory and motor $\mathrm{NCV}$ were recorded in five nerves and expressed as a cumulative index for each patient. Metabolic control was evaluated by fasting blood glucose and glycosylated haemoglobin levels. NCV index was below normal values before transplant: $-3.8 \pm 0.7$ (normal value: 0.89 ), improved 1 and 2 years after transplant: $-3.1 \pm 1.3$ and $-2.6 \pm 0.9(p=0.0019)$, stabilised until pancreas failure and deteriorated to pre-transplant values 2 years after pancreas graft failure: $-3.6 \pm 1.0$ $(p=0.034)$. Fasting blood glucose levels worsened after pancreas graft failure. $\mathrm{HbA}_{1 \mathrm{c}}$ levels, in the normal range during functioning pancreas graft $(6.6 \pm 0.6 \%)$, deteriorated after its failure $(8.0 \pm 0.6 \%, p=0.04)$. Kidney function was preserved. These data support a positive effect of pancreas transplantation per se on NCV in IDDM subjects with diabetic polyneuropathy, thus demonstrating that metabolic control provided by a self-regulated source of insulin not only halts but also ameliorates nerve function, even if polyneuropathy is advanced. [Diabetologia (1997) 40: 1110-1112]

Keywords IDDM, diabetic polyneuropathy, pancreas transplantation, kidney transplantation, metabolic control.
Diabetic polyneuropathy is a common chronic complication of diabetes mellitus, with a prevalence of $37 \%$ among insulin-dependent diabetic (IDDM) patients 18 years after disease onset [1]. Sensory and motor nerve impairment favour the development of neuropathic ulcers, which can lead to gangrene and amputations. Tight metabolic control can prevent the development of this complication, although its

Received: 14 May 1997 and in revised form: 12 June 1997

Corresponding author: S. Martinenghi, M. D., DIBIT 2A2, San Raffaele Scientific Institute, Via Olgettina 58, I-20132, Milan, Italy

A bbreviations: IDDM, Insulin-dependent diabetes mellitus; Tx, transplantation; NCV, nerve conduction velocity. effect on established diabetic polyneuropathy has not been convincingly defined, even in large cohort studies like the Diabetes Control and Complications Trial (DCCT) [1]. Moreover, several pharmacological treatments proposed when this complication is already established are inconclusive. Our study shows the effects of tight metabolic control provided by pancreas transplantation (Tx) on nerve conduction velocity $(\mathrm{NCV})$ in diabetic patients with severe diabetic polyneuropathy.

We report our findings in five IDDM patients who underwent successful kidney/pancreas Tx. Both organs functioned for at least 2 years, then pancreas graft function was lost, while kidney graft function was unaltered. Each patient served as their his/her control. 
Table 1. Nerve conduction velocity in the five nerves studied $(\mathrm{m} / \mathrm{sec})$

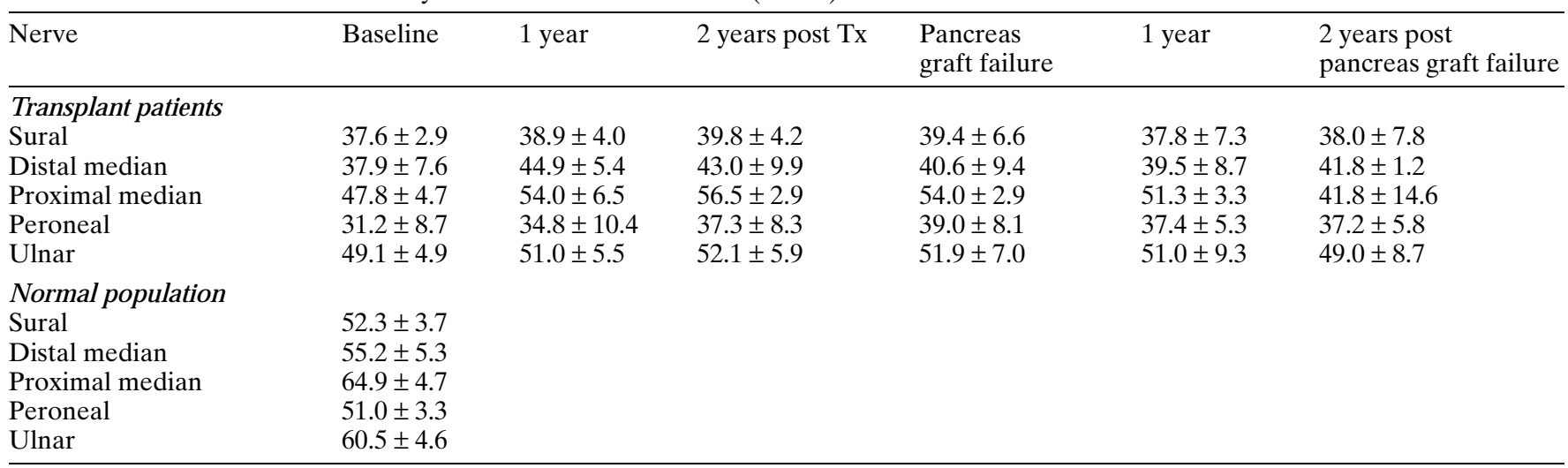

Data are mean $\pm \mathrm{SD}$

\section{Subjects and methods}

Subjects. Among 93 uraemic IDDM patients who received a kidney and pancreas Tx at our institution, 5 patients were studied. Only 5 of the 93 patients had all the characteristics necessary for the study: both pancreas and kidney grafts functioning for at least 2 years; subsequent failure of the pancreas graft only while retaining a good renal function, the kidney graft functioning for at least 2 years after pancreas failure; compliance of the patient. Age, IDDM and dialysis duration were, respectively: $37.2 \pm 5.2$ years, $24.6 \pm 5.3$ years and $13.0 \pm$ 9.0 months. All patients were affected by severe diabetic polyneuropathy and retinopathy as defined by an electrophysiological and fluorangiographic study and a clinical evaluation by a neurologist and an ophthalmologist.

M ethods: In each case a segmental, neoprene duct-injected [2] pancreas graft was performed simultaneously to a kidney Tx from a cadaveric, ABO-matched donor. Patients received the same immunosuppressive treatment (antilymphocyte immunoglobulin 4250 lymphocytotoxic units $10 \mathrm{~kg}$ body weight:over 10-14 days, Cyclosporine A $7.5-9 \mathrm{mg} \cdot \mathrm{kg}^{-1} \cdot \mathrm{day}^{-1}$, azathioprine $150 \mathrm{mg}$ /day and prednisone $1 \mathrm{mg} \cdot \mathrm{kg}^{-1} \cdot$ day $^{-1}$ tapered to $10 \mathrm{mg} /$ day). Nerve function was evaluated as NCV. Sensory NCV was recorded in the sural and in the proximal and distal median nerves, and motor NCV in the deep peroneal and ulnar nerves. Measurements were made before and at yearly intervals after Tx and before and yearly after pancreas failure. The degree of alteration of motor and sensory NCV in each patient was expressed as an index (NCV index). It was calculated by adding the deviation, expressed in SD, of each of the five nerve conduction velocity values from normal age-matched laboratory subjects and dividing the sum of these deviations by 5 [3]. The mean \pm SD for this normal population for each of the nerves studied is given in Table 1 . The normal limit in this population $(p<0.05)$ was 2 SD from the normal value, i.e. $\pm 2 \sqrt{n}$ of recorded variables, in this study \pm 0.89 . The evolution of diabetic polyneuropathy was not assessed clinically after Tx, although all patients reported a clinical improvement in neuropathic symptoms - namely numbness and paraesthesia. Fasting blood glucose, glycosylated haemoglobin $\left(\mathrm{HbA}_{1 \mathrm{c}}\right)$, serum creatinine and cyclosporine levels were recorded at the same intervals. Blood cyclosporine levels were evaluated by radioimmunoassay. Pancreas graft failure was defined clinically as a fasting blood glucose level higher than $6.6 \mathrm{mmol} / \mathrm{l}$ on repeated measurements. Pancreas graft biopsy was not performed at pancreas failure because it would have been hard to interpret in segmental, neoprene-injected pancreas.
Statistical analysis. Data were compared by the two-tailed Student's t-test; a $p$ value of 0.05 or less was considered statistically significant.

\section{Results}

All patients were insulin-independent following kidney and pancreas Tx, with normal fasting blood glucose levels and $\mathrm{HbA}_{1 \mathrm{c}}$ concentrations (see Table 2). Cyclosporine levels were in the therapeutic range throughout the study period. The pancreas graft failed $4.8 \pm 1.9$ years after Tx (range: $2-6$ ), whereas a good renal function was maintained during the whole follow-up in all patients. Functional exhaustion was postulated as the most likely cause of loss of pancreatic function in four patients; in one case anti-beta-cell autoantibodies recurred. All the patients resumed exogenous insulin therapy after pancreas failure. Despite insulin treatment, $\mathrm{HbA}_{1 \mathrm{c}}$ deteriorated after pancreas failure, probably because of the concomitant administration of steroids. Creatinine values were not significantly different before and after pancreas failure. NCV index, largely compromised before kidney and pancreas Tx, improved significantly 2 years after Tx, and stabilised until pancreas failure, although remaining far below the normal value. After pancreas failure, the NCV index rapidly deteriorated, reaching pre-Tx values 2 years after failure (Table 2).

\section{Discussion}

A functioning pancreas graft represents a self-regulated source of insulin and can restore euglycaemia and normal $\mathrm{HbA}_{1 \mathrm{c}}$ levels [4-6].

It is so far the only substitutive means of obtaining a physiologic, near-normal regulation of glucose metabolism in IDDM patients and its effects on longterm diabetic complications have been studied extensively. A positive effect of tight metabolic control attained by pancreas transplantation on diabetic 
Table 2. $\mathrm{NCV}$ index, $\mathrm{HbA}_{1 \mathrm{c}}$ and blood glucose levels following successful pancreas and kidney transplantation and after pancreas graft failure

\begin{tabular}{|c|c|c|c|c|c|c|}
\hline & Baseline & 1 year & 2 years post $T x$ & $\begin{array}{l}\text { Pancreas graft } \\
\text { failure }\end{array}$ & 1 year & $\begin{array}{l}2 \text { years post pan- } \\
\text { creas graft failure }\end{array}$ \\
\hline NCV index & $-3.8 \pm 0.7$ & $-3.1 \pm 1.3^{\mathrm{a}}$ & $-2.6 \pm 0.9^{b}$ & $-2.7 \pm 0.9$ & $-3.1 \pm 0.9$ & $-3.6 \pm 1.0^{\mathrm{d}}$ \\
\hline $\mathrm{HbA}_{1 \mathrm{c}}(\%)$ & Not available & $6.2 \pm 0.6$ & $6.5 \pm 1.1$ & $6.6 \pm 0.6$ & $8.1 \pm 0.4$ & $8.0 \pm 0.6^{\mathrm{e}}$ \\
\hline Creatinine $(\mu \mathrm{mol} / \mathrm{l})$ & Haemodialysis & $106.1 \pm 8.8$ & $97.2 \pm 8.8$ & $106.1 \pm 35.4$ & $114.9 \pm 44.2$ & $132.6 \pm 79.6$ \\
\hline $\begin{array}{l}\text { Fasting blood glucose } \\
(\mathrm{mmol} / \mathrm{l})\end{array}$ & $11.5 \pm 4.4$ & $5.4 \pm 1.8^{\mathrm{h}}$ & $4.6 \pm 0.7^{\mathrm{i}}$ & $8.7 \pm 2.8^{c}$ & $10.7 \pm 4.8^{\mathrm{f}}$ & $10.5 \pm 3.4^{\mathrm{g}}$ \\
\hline
\end{tabular}

$\mathrm{p}$ (paired Student's t-test)

${ }^{\mathrm{a}} 0.05$ vs bas, ${ }^{\mathrm{b}} 0.0019 \mathrm{vs}$ bas, ${ }^{\mathrm{c}} 0.011 \mathrm{vs} 2 \mathrm{yrs}$ post $\mathrm{Tx}$ and $0.015 \mathrm{vs}$ 1 yr post Tx, ${ }^{\mathrm{d}} 0.034$ vs $\mathrm{P}$ failure, ${ }^{\mathrm{e}} 0.04$ vs $\mathrm{P}$ failure

${ }^{\mathrm{f}} 0.005$ vs 2 yrs post $\mathrm{Tx},{ }^{\mathrm{g}} 0.03$ vs 2 yrs post $\mathrm{Tx}$

polyneuropathy has been suggested by studies comparing kidney and pancreas to kidney only grafted diabetic patients or to IDDM patients. In these reports, neuropathy showed a continuous improvement in kidney and pancreas transplanted patients. However, it has been reported that neurophysiological tests tend to worsen in a control group of IDDM nontransplanted patients, or stabilise after an initial improvement in diabetic kidney transplanted patients or show no improvement in kidney and pancreas transplanted patients with early rejection of the pancreas [3, 7-9]. In order to discriminate between the contribution of the two grafted organs on nerve function, the ideal control group would be represented by kidney and pancreas grafted patients with functioning pancreas graft but failure of the kidney transplant. In our experience, this group is difficult to study because these patients also lose their pancreas graft within a few months after kidney failure. The data observed in our small series of patients have an ideal control in that the same patients were studied before and after successful kidney and pancreas Tx and before and after pancreas failure. The pancreas graft functioned for at least 2 years, and was associated with a significant improvement of $\mathrm{HbA}_{1 \mathrm{c}}$ levels and nerve function. This improvement reached significance $(>+0.89$ SD, i.e. $p<0.05) 2$ years after $\mathrm{Tx}$ compared to baseline (1.2 SD). The magnitude of improvement in NCV is comparable to that seen in previous studies $[7,8]$, and was abrogated by pancreas graft loss, which led to a rapid decrease in NCV (0.9 SD). The consequences of pancreas loss on $\mathrm{HbA}_{1 \mathrm{c}}$ levels were significant and were paralleled by the deterioration of nerve function. The effect of the resolution of uraemia on diabetic polyneuropathy seems to be marginal, as reported in a study where a significant progression of diabetic neuropathy was observed in kidney transplanted patients with a follow-up as long as 10 years after transplantation. This suggests that uraemia plays only a small role to diabetes in the aetiology of neuropathy in uraemic diabetic subjects [10]. Despite exogenous insulin treatment, our patients were unable to maintain $\mathrm{HbA}_{1 \mathrm{c}}$ levels p (unpaired Student's t-test)

h 0.005 vs bas, ${ }^{\mathrm{i}} 0.0025$

Data are mean $\pm \mathrm{SD}, \mathrm{HbA}_{1 \mathrm{c}}$ normal range $3.5-6 \%$

within the normal range, probably because of the concomitant steroid therapy as part of the immunosuppressive treatment.

In conclusion, these findings support the concept that tight metabolic control obtained by a self-regulated source of insulin not only halts but also ameliorates nerve function, even when severe polyneuropathy is established.

\section{References}

1. The DCCT Research Group (1995) The effects of intensive diabetes therapy on the development and progression of neuropathy. Ann Int Med 122: 561-568

2. Dubernard JM, Traeger J, Neyra P, Touraine JL, Tranchant D, Blanc-Brunat N (1978) A new method of preparation of segmental pancreatic graft for transplantation: trial in dogs and in man. Surgery 84: 633-639

3. Comi G, Galardi G, Amadio S, et al. (1991) Neurophysiological study of the effect of combined kidney and pancreas transplantation on diabetic neuropathy: a 2-year follow-up evaluation. Diabetologia 34 [Suppl 1]:S103-S107

4. Sutherland DER, Najarian JS, Greenberg BZ, et al. (1981) Hormonal and metabolic effects of a pancreatic endocrine graft. Annals Int Med 95: 537-541

5. Pozza G, Bosi E, Secchi A, et al. (1985) Metabolic control of type 1 diabetes after pancreas transplantation. Br Med J 291: 510-513

6. Landgraf R, Nusser J, Riepl RL, et al. (1991) Metabolic and hormonal studies of type 1 (insulin dependent) diabetic patients after successful pancreas and kidney transplantation. Diabetologia 34 [Suppl 1]:S61-S67

7. Solders G, Tyden G, Persson A, Groth CG (1991) Improvement in diabetic neuropathy 4 years after successful pancreatic and renal transplantation. Diabetologia 34 [Suppl 1]:S125-S127

8. Kennedy WR, Navarro X, Goetz FC, Sutherland DER, Najarian JS (1990) Effects of pancreatic transplantation on diabetic neuropathy. N Engl J Med 322: 1031-1037

9. Muller-Felber W, Landgraf R, Scheuer R, et al. (1993) Diabetic neuropathy 3 years after successful pancreas and kidney transplantation. Diabetes 42: 1482-1486

10. Van Der Vliet JA, Navarro X, Kennedy WR, et al. (1988) Long-term follow-up of polyneuropathy in diabetic kidney transplant recipients. Diabetes 37(9):1247-1252 